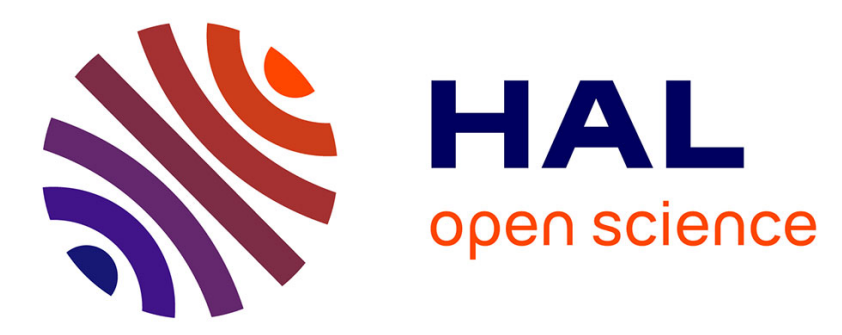

\title{
Intragranular localization induced by softening crystal plasticity analysis of slip and kink bands localization modes from high resolutionFFT-simulations results
}

\author{
A. Marano, L. Gélébart, Samuel Forest
}

\section{- To cite this version:}

A. Marano, L. Gélébart, Samuel Forest. Intragranular localization induced by softening crystal plasticity analysis of slip and kink bands localization modes from high resolutionFFT-simulations results. Acta Materialia, 2018, 175, pp.262-275. 10.1016/j.actamat.2019.06.010 . cea-02339707

\section{HAL Id: cea-02339707 https://hal-cea.archives-ouvertes.fr/cea-02339707}

Submitted on 4 Nov 2019

HAL is a multi-disciplinary open access archive for the deposit and dissemination of scientific research documents, whether they are published or not. The documents may come from teaching and research institutions in France or abroad, or from public or private research centers.
L'archive ouverte pluridisciplinaire HAL, est destinée au dépôt et à la diffusion de documents scientifiques de niveau recherche, publiés ou non, émanant des établissements d'enseignement et de recherche français ou étrangers, des laboratoires publics ou privés. 
Materialia

Elsevier Editorial system(tm) for Acta

Manuscript Draft

Manuscript Number:

Title: Intragranular localization induced by softening crystal plasticity : analysis of slip and kink bands localization modes from high resolution FFT-simulations results

Article Type: Full length article

Keywords: Crystal plasticity; Intragranular localization; Slip bands; Kink bands; FFT simulations

Corresponding Author: Dr. Lionel GELEBART, Ph.D.

Corresponding Author's Institution: CEA Saclay

First Author: Aldo Marano

Order of Authors: Aldo Marano; Lionel GELEBART, Ph.D.; Samuel Forest

Abstract: We investigate the ability of local continuum crystal plasticity theory to simulate intense slip localization observed experimentally in metals exhibiting softening mechanisms. A generic strain softening model is implemented within a massively parallel FFT solver framework to study intragranular strain localization throughout high resolution polycrystalline simulations. It is coupled to a systematic analysis strain localization modes: Equivalent plastic strain and lattice rotation fields are processed to create binary maps of slip and kink bands populations, estimate their volume fraction and mean strain level. High resolution simulations show the formation of an intragranular localization band network. The associated localization maps are used to identify accurately slip and kink bands populations and highlight the distinct evolution of kink bands, influenced by lattice rotation. It is evidenced that selection between slip or kink localization modes is only due to grain to grain incompatibilities with classical crystal plasticity models, as they do not account for their actual physical differences. As a result they predict the formation of a large amount of kink bands, whereas experimental observations of strongly softening metals (such as irradiated metals) seldom report them.

Suggested Reviewers: Ricardo Lebensohn

Materials Science and Technology Division, Los Alamos National Laboratory lebensodlanl.gov

R. Lebensohn has worked extensively on full-field FFT based method simulations for polycrystal behavior modelling, and worked on kink banding in ice polycrystals.

Jeffrey Kysar

Chair Professor, Department of Mechanical Engineering, Columbia University

jk2079@columbia.edu

Pr. Kysar work include experimental observations of kink bands in single crystals and continuum crystal plasticity simulations. 
David McDowell

Georgia Tech

david.mcdowell@me.gatech.edu

Our paper is in direct relation with Pr. McDowell's work on plastic flow localization in irradiated polycrystal modeling.

Fionn Dunne

Imperial College London

fionn.dunnedimperial.ac.uk

Our paper is in direct relation with Pr. Dunne's work on plastic flow localization in irradiated polycrystals modeling. 
Lionel GELEBART

Commissariat à l'Energie Atomique

Centre de Saclay

DMN/SRMA/LC2M - PC 46

91191 Gif-sur-Yvette Cedex France

Phn: +33169081678

Fax: +33169087167

E-Mail: lionel.gelebart@cea.fr

Dear editors,

Please find attached our manuscript entitled « Intragranular localization induced by softening crystal plasticity: Analysis of slip and kink bands localization modes from high resolution FFT-simulations results », to be considered for publication in Acta Materialia.

This paper investigates the ability of continuum crystal plasticity to simulate intragranular plastic slip localization in polycrystals exhibiting softening mechanisms. The originality of the paper is to analyze simulated localization bands nature (slip or kink bands). To this end, we developed a post-processing method to produce a systematic analysis of slip and kink band networks from full-field simulations. We show that both modes are activated whereas kink bands are not observed experimentally, for instance in irradiated polycrystals. A general discussion on the fundamental reasons of localization modes formation with classical crystal plasticity models concludes this work.

Thank you for your consideration of this manuscript.

Best regards,

A.Marano, L.Gélébart, S. Forest 


\begin{abstract}
We investigate the ability of local continuum crystal plasticity theory to simulate intense slip localization observed experimentally in metals exhibiting softening mechanisms. A generic strain softening model is implemented within a massively parallel FFT solver framework to study intragranular strain localization throughout high resolution polycrystalline simulations. It is coupled to a systematic analysis strain localization modes: Equivalent plastic strain and lattice rotation fields are processed to create binary maps of slip and kink bands populations, estimate their volume fraction and mean strain level. High resolution simulations show the formation of an intragranular localization band network. The associated localization maps are used to identify accurately slip and kink bands populations and highlight the distinct evolution of kink bands, influenced by lattice rotation. It is evidenced that selection between slip or kink localization modes is only due to grain to grain incompatibilities with classical crystal plasticity models, as they do not account for their actual physical differences. As a result they predict the formation of a large amount of kink bands, whereas experimental observations of strongly softening metals (such as irradiated metals) seldom report them.
\end{abstract}

Keywords: Crystal plasticity, Intragranular localization, Slip bands, Kink bands, FFT simulations 


\section{Introduction}

A critical issue to address in bridging the gap between microscopic mechanisms and macroscopic mechanical behavior of crystalline materials is the intrinsically heterogeneous nature of plastic slip. Several authors have provided a precise description of these discrete 5 phenomena $[1,2]$ : deformation occurs by formation of discrete surface steps caused by the emergence of dislocations. When intense dislocation glide occurs on a few crystallographic planes, a sharp slip localization band parallel to dislocations glide planes forms called slip band, widely observed in metallic single crystals and polycrystals. Another type of slip localization band observed in deformed crystals, associated with high lattice rotation and orthogonal to the glide direction, is the so-called kink band. Kink bands are reported as a late deformation mode of metallic single crystals $[1,3]$, in strongly anisotropic hexagonal crystals such as ice or Zinc $[4,5,6,7,8]$, as a crack-tip localization mode $[9,10,11]$ or for titanium alloys under high strain rate deformation [12]. Asaro and Rice bifurcation analysis [13] showed that in presence of strain softening the constitutive equations of crystal plasticity 15 can predict both localization modes, and a few authors have studied the formation of kink bands in crystal plasticity simulations $[14,15,16,5]$.

Theoretical and numerical studies have evidenced the role of local softening mechanisms in the apparition of heterogeneous deformation $[17,18]$. These mechanisms result of interactions between dislocations and crystal defects and are particularly intense in several metals such as irradiated or hydrogen charged polycrystals. In that case dislocation sources are locked by a hydrogen atom atmosphere making their activation much harder. Therefore required stress to generate further slip from an already active source is thought to be lower than stress needed to activate a new locked source thus promoting intense slip on a limited number of atomic planes [19,20]. Another example is the extensively studied dislocation channeling mechanism observed in a large variety of irradiated metals such as Copper [21, 22], Zirconium [23, 24], steel [25, 26, 27, 28] or Vanadium [29], but also quench-hardened

\footnotetext{
*Corresponding author

Email addresses: aldo.marano@cea.fr (Aldo Marano), lionel.gelebart@cea.fr (Lionel Gélébart), samuel.forest@mines-paristech.fr (Samuel Forest)
} 
aluminum [30] and gold [31] single crystals. Those materials are hardened by a high density of nanometer size defects like dislocation loops or stacking fault tetrahedra. Their interaction with gliding dislocations leads to their progressive sweeping/annihilation [32] and so thus to a strong local softening promoting further slip in the region where it has already occurred. Defect-free channels parallel to dislocation glide planes are indeed observed after deforming those materials, and are strongly correlated to surface slip steps indicating that defect-annihilation based softening is responsible for the formation of intense slip bands. A similar mechanism is observed in $\omega$-enriched Ti-Nb-based gum metals where $\omega$-particles depleted channel are correlated to surface localization bands [33]. Conversely correlation of clear channels with kink bands has never been observed and more generally kink bands are not reported as intragranular localization modes in strongly softening crystals.

Numerous efforts have been made aiming at modeling these phenomenon at the polycrystal scale as they have a first order influence on the macroscopic mechanical behavior or on cracking initiation due to localization induced stress concentrations. Relying on molecular dynamics or dislocations dynamics analyses of the dislocation channeling mechanism [34, 35, 36], many dislocation-based classical crystal plasticity models have been developed to simulate irradiated metals behavior [37, 38, 39, 40, 41, 42, 43], accounting for the softening through local defect-density dependent critical shear stress and defect-density evolution equation modeling their annihilation with increasing glide. This framework successfully reproduces the main features of macroscopic behavior of irradiated metals.

These strain softening based models have also been used to conduct full-field simulations of strain localization. Sauzay et al. [25] have explicitly modeled one or two predefined soft clear channels embedded in a hard matrix in order to study induced stress concentrations. Zhang et al. [44] have followed a different approach at the polycrystal scale by prescribing softening only on a predefined network of potential slip bands in 2D polycrystalline FE simulations. This modeling strategy allows localization bands to form but requires prior knowledge of geometric characteristics of the bands network. On the contrary, Erinosho and Dunne [45] have modeled a full 3D polycrystal with a strain softening behavior with 

lar localization bands due to slip softening, however they used cubic grains microstructure and associated mesh resolution (average of 1000 elements per grain) is too coarse to reproduce accurately intragranular slip localization. Patra and McDowell [46, 47] have recently published simulations with higher resolution using a dislocation channeling based softening model, and their results predict indeed formation of intragranular localization bands. However they simulated a 2D aggregate under the generalized plane strain hypothesis which is not representative of complex localization patterns that can arise in 3D microstructures. Most importanty, they did not analyze the slip or kink nature of the simulated bands in order to compare results with experimentally observed cleared channels in irradiated steels.

This work aims at gaining deeper insight into the ability of softening classical crystal plasticity to simulate accurately intense intragranular localization observed in the various metals exhibiting softening mechanisms. To this end, we implemented a generic softening crystal plasticity model within a massively parallel FFT solver allowing to model three dimensional polycrystalline unit cells with higher grid resolutions and number of grains. A systematic analysis of simulated localization bands is carried out on simulations results. For that purpose, we developed a methodology using full-field outputs of the FFT simulations to build localization mode maps that allow to identify and quantify simulated slip and kink bands populations.

\section{Crystal plasticity constitutive model}

The constitutive model used in this study reduces to the two main ingredients allowing to simulate strain localization along crystallographic planes: the classical finite deformation crystal plasticity framework combined with a softening flow rule. This simple and generic formulation allows to avoid any material complexity to focus the study on the link between strain softening crystal plasticity and slip localization. Crystal plasticity kinematics is described by the classical multiplicative decomposition of the deformation gradient tensor $\boldsymbol{F}$ into its elastic part $\boldsymbol{F}_{\boldsymbol{e}}$ and its plastic part $\boldsymbol{F}_{\boldsymbol{p}}[48]: \boldsymbol{F}=\boldsymbol{F}_{\boldsymbol{e}} \cdot \boldsymbol{F}_{\boldsymbol{p}}$. where $\boldsymbol{F}_{\boldsymbol{p}}$ maps the 
reference configuration to the isoclinic stress-free local configuration where crystal lattice orientation is unchanged with respect to the reference configuration and $\boldsymbol{F}_{\boldsymbol{e}}$ maps the intermediate local configuration into the deformed configuration, describing crystal lattice distortion and rotation. It is assumed that plastic deformation takes place through the slip of dislocations on prescribed slip planes with normal $\boldsymbol{n}^{\alpha}$ along slip direction $\boldsymbol{m}^{\alpha}$. The plastic velocity gradient $\boldsymbol{L}_{p}=\dot{\boldsymbol{F}}_{\boldsymbol{p}} \cdot \boldsymbol{F}_{\boldsymbol{p}}^{-1}$ is then determined by the shearing rate of the $N_{s}$ material slip systems through the relation :

$\boldsymbol{L}_{p}=\sum_{s}^{N_{s}} \dot{\gamma}^{\alpha} \boldsymbol{\mu}^{\alpha}$

where $\gamma^{\alpha}$ is the plastic slip on slip system $\alpha$ and $\boldsymbol{\mu}^{\alpha}=\boldsymbol{m}^{\alpha} \otimes \boldsymbol{n}^{\alpha}$ the Schmid tensor for slip system $\alpha$. Crystal elasticity is defined by a linear relation between the Green-Lagrange elastic strain tensor $\boldsymbol{E}_{e}=\frac{1}{2}\left(\boldsymbol{F}_{e}^{T} \cdot \boldsymbol{F}_{\boldsymbol{e}}-\mathbf{1}\right)$ and Piola elastic stress tensor $\boldsymbol{\Pi}_{e}=\operatorname{det}\left(\boldsymbol{F}_{\boldsymbol{e}}\right) \boldsymbol{F}_{\boldsymbol{e}}^{-1} \cdot \boldsymbol{\sigma} \cdot \boldsymbol{F}_{\boldsymbol{e}}^{-T}$ (defined in the isoclinic configuration) : $\boldsymbol{\Pi}_{e}=\underline{\underline{\boldsymbol{\Lambda}}}: \boldsymbol{E}_{e} \cdot \underline{\underline{\boldsymbol{\Lambda}}}$ is the fourth-order elastic tensor, $\boldsymbol{\sigma}$ the Cauchy stress tensor w.r.t. current configuration. Plastic flow is described by a Nortontype visco-plastic flow rule (Eq. 2) and an exponential softening of critical resolved shear stress $\tau_{c}^{\alpha}$ with increasing cumulated slip on each slip system $\gamma_{c u m}^{\alpha}$ (Eq. 4).

$\dot{\gamma}^{\alpha}=\left\langle\frac{\left|\tau^{\alpha}\right|-\tau_{c}^{\alpha}}{K}\right\rangle^{n} \operatorname{sgn}\left(\tau^{\alpha}\right)$

$\tau^{\alpha}=M: \boldsymbol{\mu}^{\alpha}$

$\tau_{c}^{\alpha}=\tau_{c_{i}}^{\alpha}-\Delta \tau^{\alpha}\left(1-\exp \left(-\frac{\gamma_{c u m}^{\alpha}}{\gamma_{0}^{\alpha}}\right)\right)$

Resolved shear stress $\tau^{\alpha}$, is calculated by the projection of the Mandel stress tensor $\boldsymbol{M}=$ $\operatorname{det}\left(\boldsymbol{F}_{\boldsymbol{e}}\right) \boldsymbol{F}_{\boldsymbol{e}}^{T} \cdot \boldsymbol{\sigma} \cdot \boldsymbol{F}_{\boldsymbol{e}}^{-T}$ on slip system $\alpha$ (Eq. 3). $n$ and $K$ are Norton-law parameters. The model involves three important material coefficients : $\tau_{c_{i}}, \Delta \tau^{\alpha}$ are respectively the initial critical shear stress and the maximum softening that can be reached on slip system $\alpha$, and $\gamma_{0}^{\alpha}$ is a parameter adjusting the softening rate.

Despite its simplicity, we believe it to be representative of most softening models for irradiated metals $[37,38,39,40,41,42,43,49]$. They are indeed formulated within the same kinematic framework and rely on softening rules that, when written in the case of 
single slip, reduce to the form :

$$
\begin{gathered}
\tau_{c}=\tau_{0}+A \sqrt{\rho_{D}} \\
\dot{\rho}_{D}=-B \rho_{D}|\dot{\gamma}|
\end{gathered}
$$

$A$ and $B$ being material coefficients and $\rho_{D}$ a defect density. Eq. 5 describes hardening due to local defect density and Eq. 6 defect sweeping by gliding dislocations, inducing softening. When integrated for a constant shear rate these equations yield an exponential decay of the critical resolved shear stress similar to Eq. 4.

\section{Slip/kink localization modes analysis}

In this section, we present a post processing methodology designed to identify the nature of localization bands in FFT simulations results. In order to identify localization bands, we use the equivalent plastic strain field defined by Eq. 8 as a measure of local slip intensity. Contrary to slip bands, kink bands involve high lattice rotation that can be used to distinguish kinks from slip bands. Lattice rotation angle is computed using the polar decomposition of the elastic part of the deformation gradient : $\boldsymbol{F}_{\boldsymbol{e}}=\boldsymbol{R}_{e} \cdot \boldsymbol{U}_{e}$. Neglecting the small elastic distortion described by the right stretch tensor $\boldsymbol{U}_{e}$, the elastic rotation tensor $\boldsymbol{R}_{e}$ is interpreted as the lattice rotation and the corresponding angle $\theta$ can be computed with Eq. 7. $p$ is the cumulative plastic strain.

$$
\begin{aligned}
& \theta=\arccos \left(\frac{1}{2}\left(\operatorname{tr}\left(\boldsymbol{R}^{e}\right)-1\right)\right) \\
& p=\int_{0}^{t} \sqrt{\boldsymbol{L}_{p}: \boldsymbol{L}_{p}} d t
\end{aligned}
$$

These fields are then used to define $L$ and $R$, indicator functions respectively of slip localization and high lattice rotation areas, by the relations:

$$
\begin{aligned}
L(\boldsymbol{X}) & =\mathcal{H}\left(P(\boldsymbol{X})-\bar{p} \Phi_{D}\right) \\
R(\boldsymbol{X}) & =\mathcal{H}\left(\theta(\boldsymbol{X})-\bar{\theta} \Phi_{R}\right)
\end{aligned}
$$

where $\mathcal{H}$ is the Heaviside step function and $\boldsymbol{X}$ is the material point coordinate vector. They indicate regions where fields $p$ and $\theta$ are above a level defined by their mean value $\bar{p}$ and 
$\bar{\theta}$ over the whole unit cell multiplied by suitably chosen relative thresholds, $\Phi_{D}$ and $\Phi_{R}$ respectively. Therefore $L$ maps plastic strain localization areas that are mainly localization bands. As mentioned above, kink bands can be distinguished from slip bands because they involve large lattice rotation which suggests a natural definition of kink bands as areas exhibiting both intense plastic slip and high lattice rotation, that is to say $L(\boldsymbol{X})=1$ and $R(\boldsymbol{X})=1$. We then assume that localization area without intense lattice rotation are slip bands. Hence, kink and slip bands indicator functions, $S$ and $K$, are given by:

$$
\begin{aligned}
K(\boldsymbol{X}) & =L(\boldsymbol{X}) \cdot R(\boldsymbol{X}) \\
S(\boldsymbol{X}) & =L(\boldsymbol{X})-K(\boldsymbol{X})
\end{aligned}
$$

and can be plotted simultaneously to evidence localization modes. Finally, these functions are used to determine for the slip and kink bands population, volume fractions, $f_{S}$ and $f_{K}$, and mean value of equivalent plastic strain, $\left\langle P_{S}\right\rangle$ and $\left\langle P_{K}\right\rangle$, as follows :

$$
\begin{aligned}
& <P_{K}>=\frac{1}{f_{K} V} \int_{\Omega} K(\boldsymbol{X}) P(\boldsymbol{X}) \boldsymbol{d} \boldsymbol{X} \\
& <P_{S}>=\frac{1}{f_{S} V} \int_{\Omega} S(\boldsymbol{X}) P(\boldsymbol{X}) \boldsymbol{d} \boldsymbol{X}
\end{aligned}
$$

\section{FFT Simulations}

Simulations are performed using the FFT solver AMITEX_FFTP ${ }^{1}$ which offers two main advantages. First, its massively parallel implementation enables to simulate strain localization in three dimensional and high resolution polycrystalline unit cells in order to overcome limitations observed in the literature. Previous simulations aiming at modeling softening induced strain localization where indeed conducted either on 3D microstructures but with low resolutions [45] or with high resolution but 2D microstructures [47]. Second, FFT based methods provide mechanical fields in the form of 3D images very well suited for the computation of localization modes indicator functions and associated quantities defined in the former section.

\footnotetext{
${ }^{1}$ http://www.maisondelasimulation.fr/projects/amitex/html/
} 


\subsection{Numerical implementation}

The FFT solver AMITEX is based on the original fixed-point numerical scheme proposed by Moulinec and Suquet [50] but implements a modified discrete Green operator based on a finite difference method to evaluate derivatives in Fourier space, equivalent to the one proposed by Willot [51]. This numerical scheme is shown to be equivalent to the use of hexahedral finite elements with reduced integration [52]. In addition, Anderson's convergence acceleration technique is applied to the fixed point algorithm [53, 54, 55].

Material constitutive law is integrated using a fully implicit $\theta$-method implementation of constitutive equations generated by the MFront code generator [56]. Internal variables increments $\left\{\Delta \boldsymbol{E}_{e}, \Delta \gamma^{\alpha}\right\}$ between time $t$ and $t+\Delta t$ are computed from their value at time $t$ $\left\{\Delta \boldsymbol{E}_{e}^{t}, \gamma^{\alpha, t}\right\}$ by solving with Newton-Raphson algorithm the following system of non linear equations :

$$
\begin{array}{r}
\Delta \boldsymbol{E}_{e}+\boldsymbol{E}_{e}^{t}-\frac{1}{2}\left(\boldsymbol{F}_{\boldsymbol{e}}^{T, t} \cdot \boldsymbol{F}_{\boldsymbol{e}}^{t}-\mathbf{1}\right)=0 \\
\Delta \gamma^{\alpha}-\left\langle\frac{\left|\tau^{\alpha}\right|-\tau_{c}^{\alpha}}{K}\right\rangle^{n} \operatorname{sgn}\left(\tau^{\alpha}\right) \Delta t=0
\end{array}
$$

Note that here Green-Lagrange elastic strain $\boldsymbol{E}_{e}$ tensor is considered as an additional variable to avoid inaccuracies in numerical evaluation of $\boldsymbol{F}_{\boldsymbol{e}}$, following the approach presented in Ling et al. [57].

\subsection{Simulations description}

FFT simulations apply periodic boundary conditions to 3D regular grids of voxels (i.e. 3D images). Two types of polycrystalline microstructures have been generated using voxelized periodic Voronoi tessellations :

- 2D periodic unit cells (1 voxel thick in the $X$ direction) containing 225 grains $\left(15^{2}\right)$. Because of $X$ direction periodicity, this is equivalent to 3D infinite columnar grains in the $X$ direction, also equivalent to a 2D modeling under generalized plane strain hypothesis. Three in plane slip systems with a 60 degree misorientation relative angle are modeled ( Fig. 1), and each grain is assign a random "2D orientation" is assigned to each grain, leaving all slip plane normals and glide directions in the $(Y, Z)$ plane. 


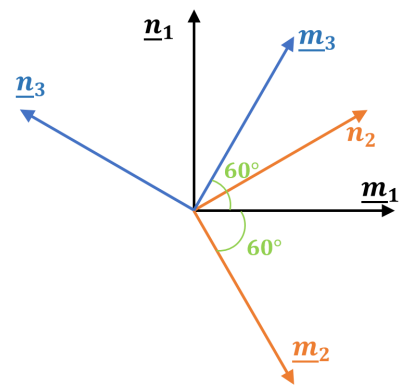

Figure 1: Three inplane coplanar slip systems used for 2D simulations on flow rule parameter is omitted in the rest of this paper. The single slip system softening behavior and simulated macroscopic mechanical behavior for 2D, 3D FCC and 3D BCC polycristals are plotted on Fig. 2 for this set of material parameters which involves $20 \%$ maximum softening of the critical resolved shear stress on each slip system. 


\begin{tabular}{|c|c|c|c|c|c|c|}
\hline $\mathrm{K}$ & $\mathrm{n}$ & $\mathrm{E}$ & $\nu$ & $\tau_{C_{i}}$ & $\tau_{C_{f}}$ & $\gamma_{0}$ \\
\hline $10 \mathrm{MPa} . \mathrm{s}^{\frac{1}{n}}$ & 15 & $100000 \mathrm{MPa}$ & 0.3 & $100 \mathrm{MPa}$ & $80 \mathrm{MPa}$ & 0.1 \\
\hline
\end{tabular}

Table 1: Material parameters used for all simulations

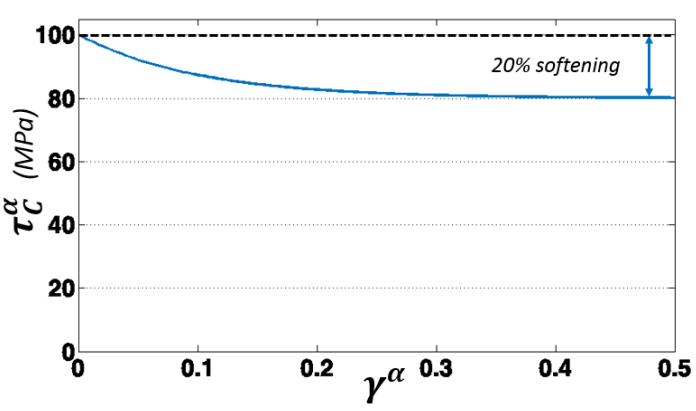

(a)

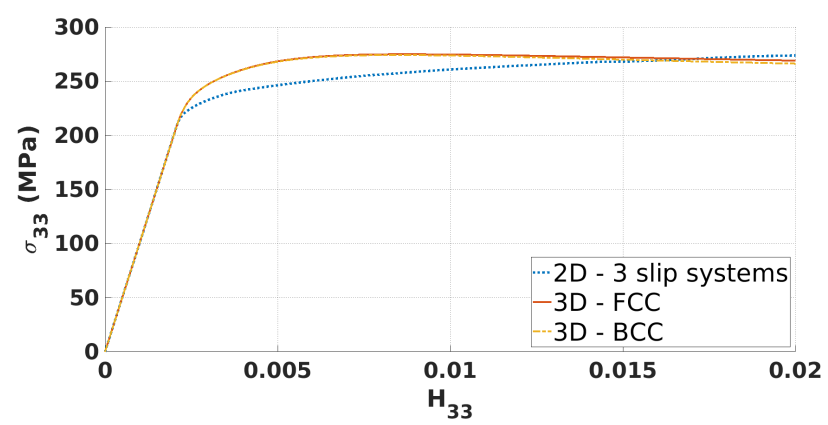

(b)

Figure 2: Critical resolved shear stress evolution curve used for all simulations (a), and associated macroscopic stress-strain responses for different polycrystalline simulations (b) (FCC and BCC curves are almost superimposed)

\section{Results}

\subsection{Identification of slip and kink bands}

Fig. 3 (a-b) shows equivalent plastic strain and lattice rotation fields simulated for the 2D microstructure after $1 \%$ overall elongation. Clear networks of intragranular slip localization bands have formed as well as intense lattice rotation bands. Indicator functions $S$ and $K$ of slip/kink bands are computed from these fields as defined in sec. 3. They are plotted respectively in red/blue and superposed to the microstructure in order to construct the associated localization mode map (c). In addition, slip planes traces are superposed to compare the detected slip and kink band orientations to crystallographic directions. The zoomed view (d) clearly demonstrates that all red/blue bands are respectively parallel/perpendicular to a slip plane. Fig. 4 shows similar results for a 3D simulation of a FCC polycrystal, as well as the video provided as supplementary material.

A systematic study of simulated bands confirms that this correspondence holds for most detected bands. The proposed methodology provides then an efficient tool to identify plastic 


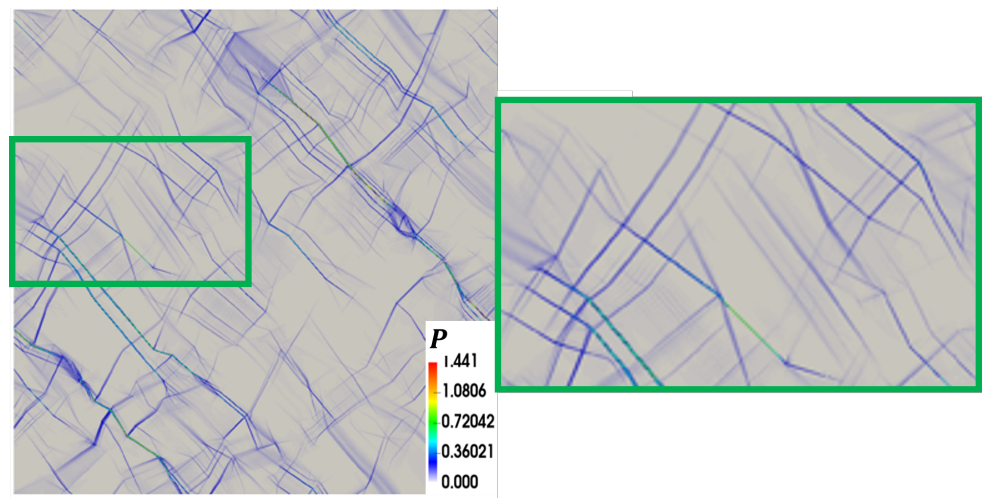

(a)

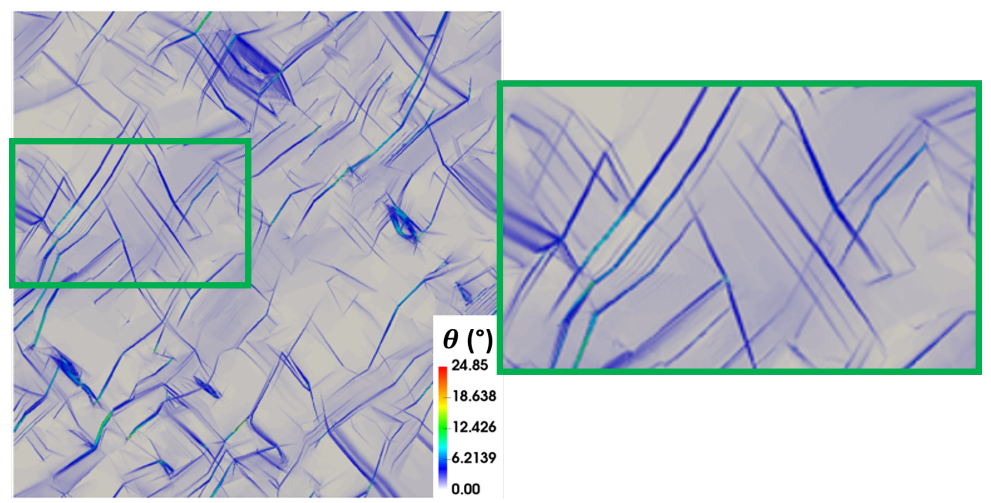

(b)

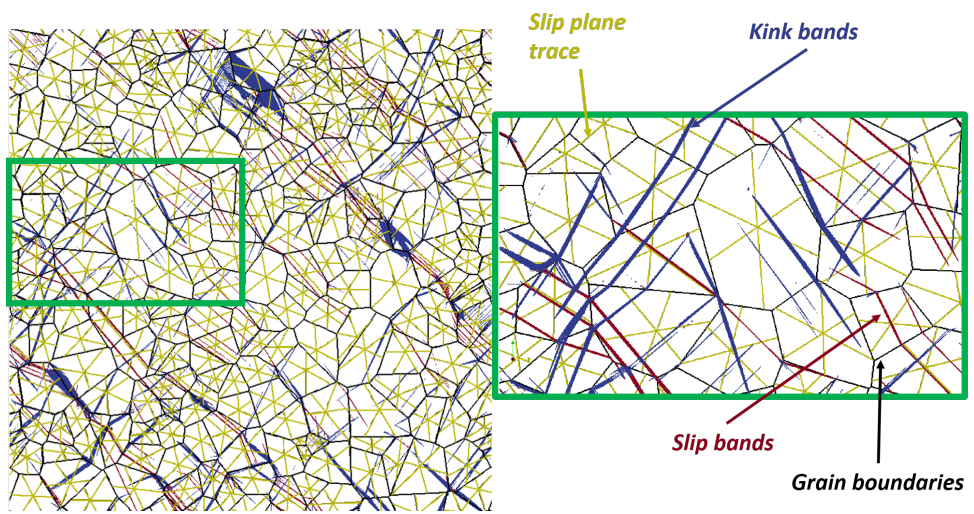

(c)

(d)

Figure 3: Equivalent plastic strain (a) and lattice rotation angle (b) fields for the 2D polycrystal (3 inplane slip systems) after $1 \%$ overall strain. Associated localization map (c). Slip (red) and kink (blue) bands are always respectively parallel/orthogonal to a slip plane (yellow) as illustrated by the zoom at the green-surrounded region (d). Grain boundaries are represented by black lines. Grid resolution: 750x750 voxels 

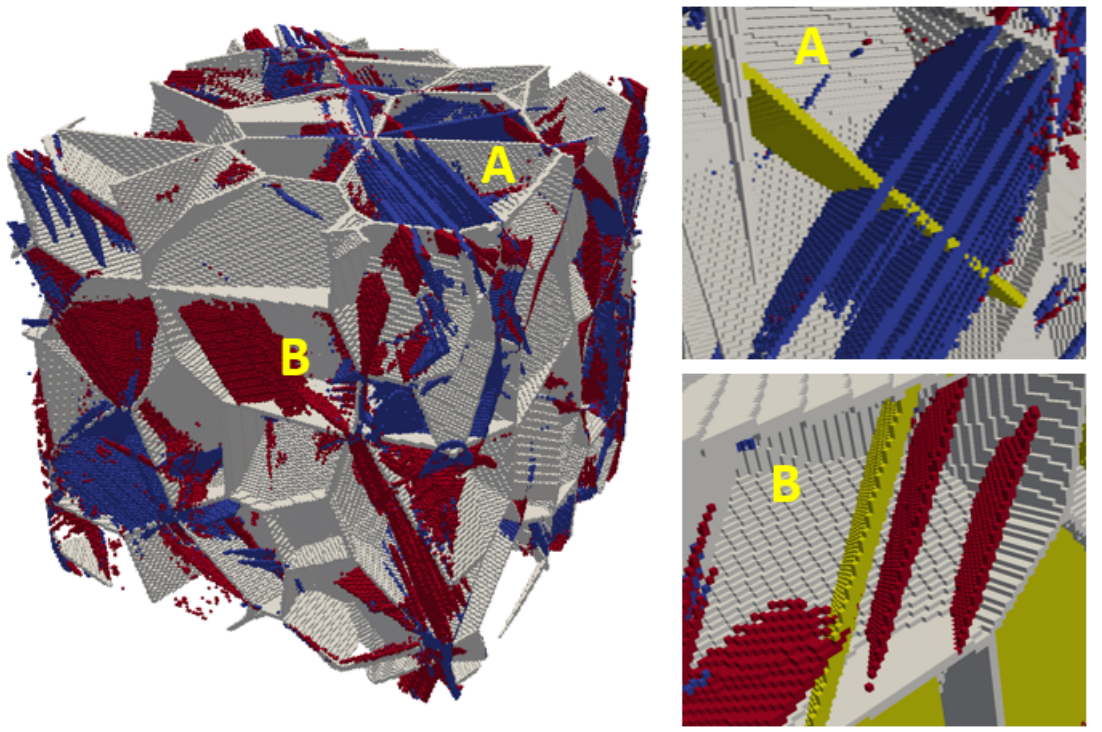

Figure 4: Localization map built for a FCC 3D polycrystal after $2 \%$ overall strain showing slip/kink bands (red/blue) and grain boundaries (grey). Activated slip plane (yellow) is plotted on the zooms on A and B marked grains. The A grain contains a series of kink bands orthogonal to the activated slip plane. The B grain contains 2 slip bands parallel to the activated slip plane. Grid resolution: 200x200x200 voxels

localization modes that could be applied to any finite strain crystal plasticity based simulation. It is especially helpful to analyze 3D simulations (see. Fig. 4) which lead to complex 3D images of dense planar bands network, making direct observation of bands orientation cumbersome.

Thresholds detection values have been tuned by hand to obtain optimal maps. Too low values of $\Phi_{D}$ leads to detection of more homogeneously deformed area whereas too high values leads to the detection of only a few localization bands. Best compromise has been achieved for $\Phi_{D}=3 . \Phi_{R}$ is then chosen to obtain best optimal band separation. Too high values lead to identification of kinks exhibiting too low lattice rotation as slips. Besides, regions with a slight inhomogeneous deformation can also have a moderate local lattice rotation and a slip band crossing them would be identified as a kink for too low values of $\Phi_{R}$. Optimal results have been obtained for $\Phi_{R}=2$. Thresholds values do not have a strong impact on qualitative analysis of localization maps. However they have a stronger influence on bands volume fraction and mean plastic strain estimation. Yet using the same 


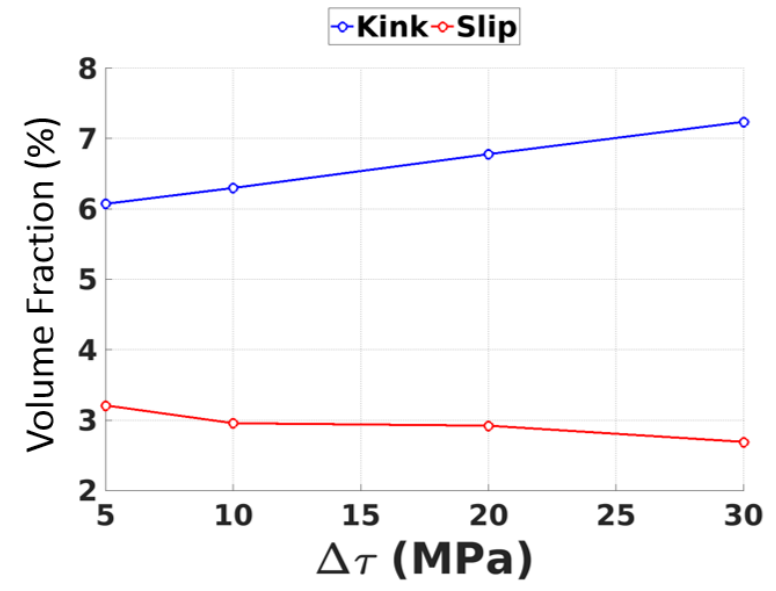

(a)

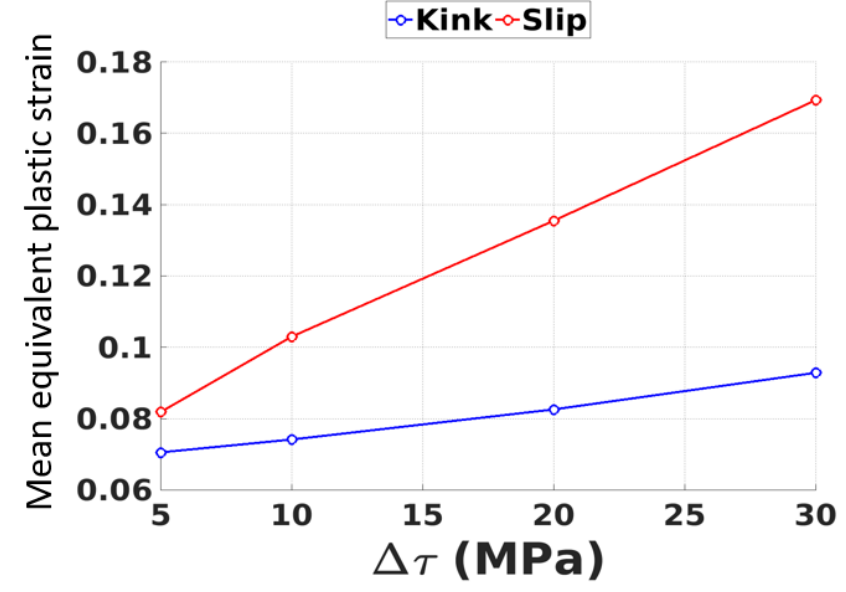

(b)

Figure 5: Evolution of slip/kink bands volume fraction (a) and mean equivalent plastic strain (b) with softening intensity for the 2D polycrystal (3 in-plane slip systems) after 1\% overall strain (slip/kink bands represented in red/blue respectively). Grid resolution: 750x750 voxels.

set of threshold values to analyze different simulations allows for relative comparison of these quantities that provides qualitative insights on strain localization properties, as presented 165 in the following sections.

\subsection{Grid resolution influence on slip and kink bands}

Fig. 6 shows the evolution of slip and kink band volume fractions and mean plastic strain after $1 \%$ total elongation for 2D simulations conducted with increasing grid resolution. When increasing grid resolution, slip bands volume fraction decreases and their mean strain level increases. Softening material behavior is known to induce numerical instabilities leading to such mesh dependence. On the contrary, results show that kink bands properties are much less sensitive to grid resolution and seem to converge. Associated localization maps illustrate this trend: When increasing grid resolution the slip bands thickness decreases while their number increases. In contrast kink bands patterns are very similar in the three maps.

Due to lattice rotation, the Schmid factor in kink bands can be locally decreased (or the reverse) and hinder further slip. Hence, lattice rotation hardening restrain slip localization. This rotation induced hardening mechanism opposes to material softening and can explain 
why kink bands population exhibit a low mesh size dependance despite the strongly softening material behavior.

\subsection{Softening influence on slip and kink bands}

Fig. 5 presents evolution of slip/kink volume fractions and mean plastic strain after $1 \%$ total elongation when varying the maximum softening level $\Delta \tau$ for $2 \mathrm{D}$ simulations. Again, slip and kink band population evolutions present significant differences. Increasing $\Delta \tau$ causes slip bands volume fraction to slightly decreases whereas kink bands volume fraction increases. The mean plastic strain level increases with softening intensity for both populations but increase is steeper for slip bands.

Softening will also promote increased plastic slip in kink bands but because of rotation induced hardening, slip localization will be hindered and additional plastic slip will occur by widening of existing kink bands or formation of new ones. This mechanism explains the smaller increase in kink bands mean plastic strain level as well as their increased volume fraction for a higher $\Delta \tau$.

\subsection{Comparison between $2 D$ and $3 D$ simulations}

Fig. 7 and Fig. 8 show the evolution of slip/kink band volume fractions and mean plastic strain level with increasing loading for a $2 \mathrm{D}$ simulation and a $3 \mathrm{D}$ simulation of a FCC polycrystal. Results highlight a clearly distinct evolution of slip and kink band populations for the 2D simulations. Both modes are present in comparable amounts in the first stages of deformation but then volume fraction grows more quickly for kinks than slip bands. Localization map snapshots on Fig. 7 (a) provide additional information on slip and kink population evolution: Kink bands volume fraction increases due to both band formation and existing band widening whereas only the first phenomenon is observed for slip bands. Conversely slip and kink band evolutions are much closer in the 3D case and no clear difference in band thickness is observed on localization map snapshots Fig. 7 (b). Likewise Fig. 8 evidences a clear gap between slip/kink mean plastic strain level in the 2D simulation while they are almost identical in the 3D case. 


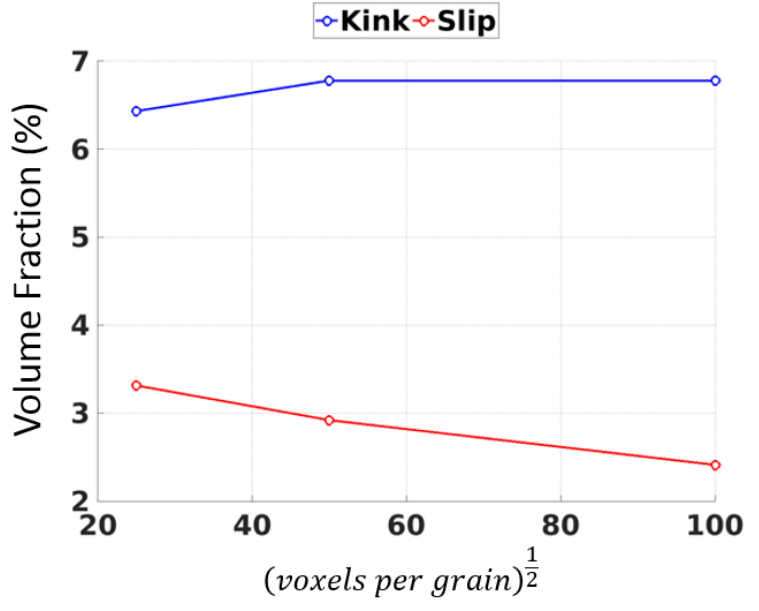

(a)

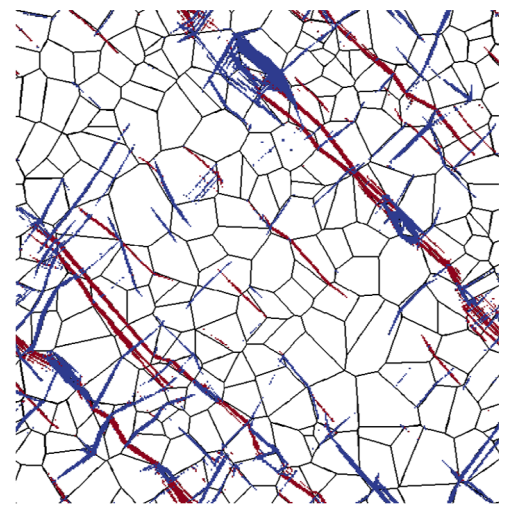

(c) $25^{2}$ voxels per grain

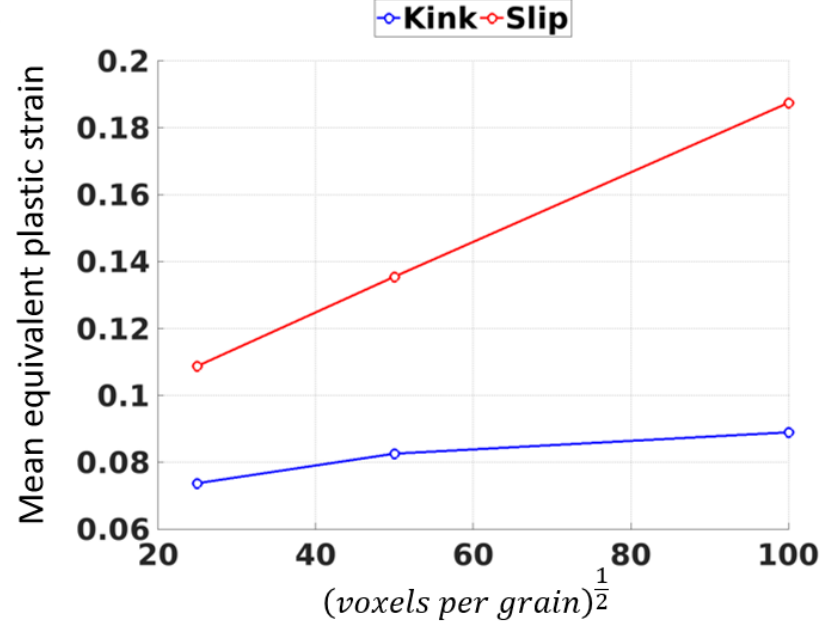

(b)
29

30

31

32

33

34

35

36

37

38

39

40

41

42

43

44

45

46

47

48

49

50

51

52

53

54

55

56

57

58

59

60

61

62

63

64

65

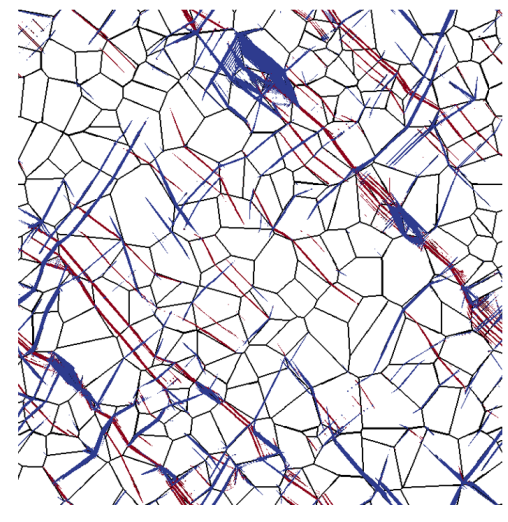

(d) $50^{2}$ voxels per grain

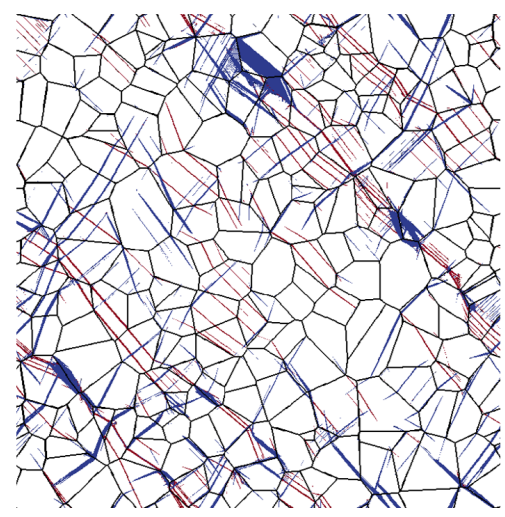

(e) $100^{2}$ voxels per grain

Figure 6: Influence of grid resolution on slip (red) and kink (blue) bands volume fraction (a) and mean equivalent plastic strain (b) for the 3 in-plane slip systems 2D polycristal after $1 \%$ overall strain. (c - e): associated localization maps. 


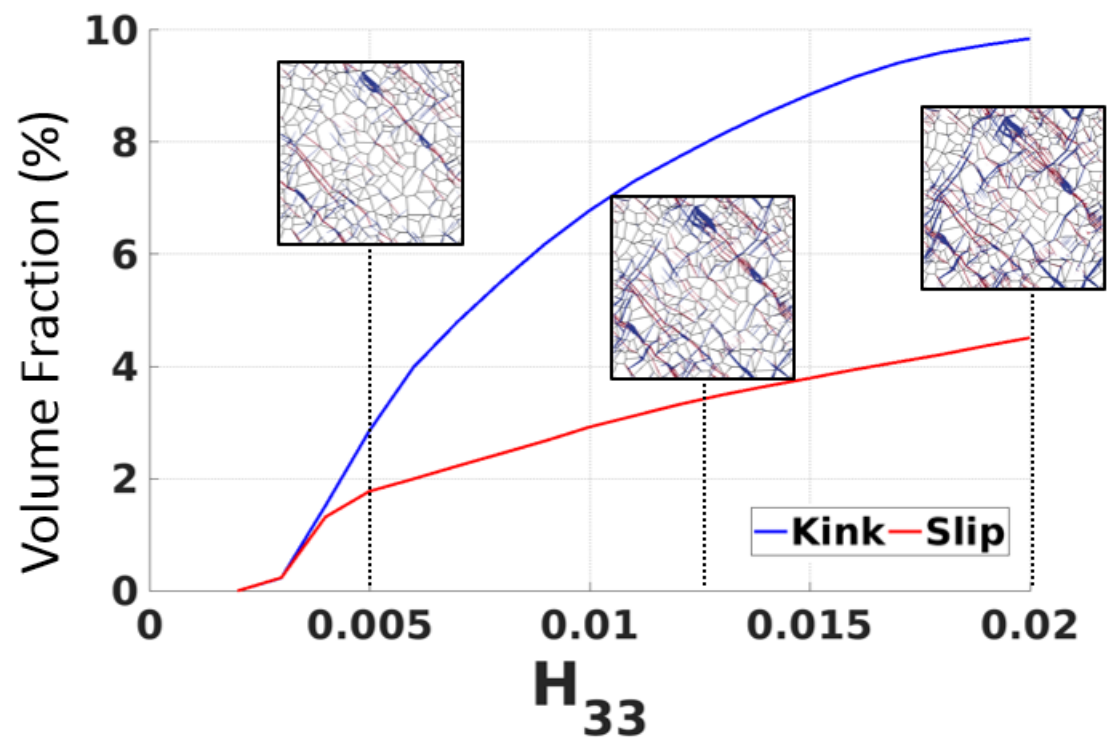

(a)

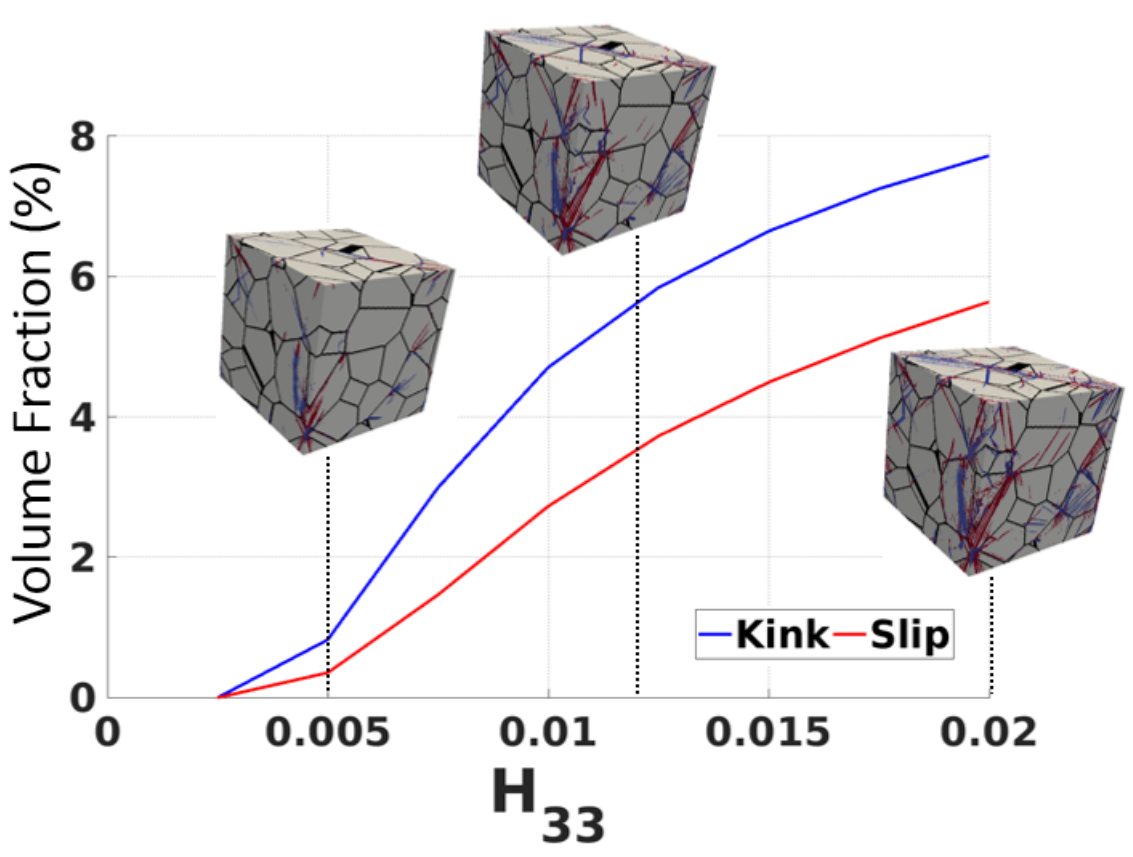

(b)

Figure 7: Evolution of slip/kink band volume fraction for the 2D simulation with 3 in-plane slip system (a) and a FCC 3D polycrystal (b), with snapshots of associated localization maps. 


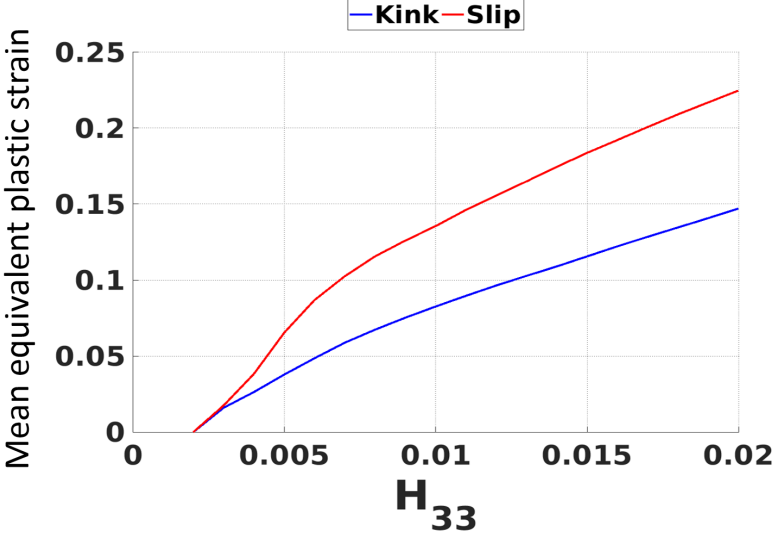

(a)

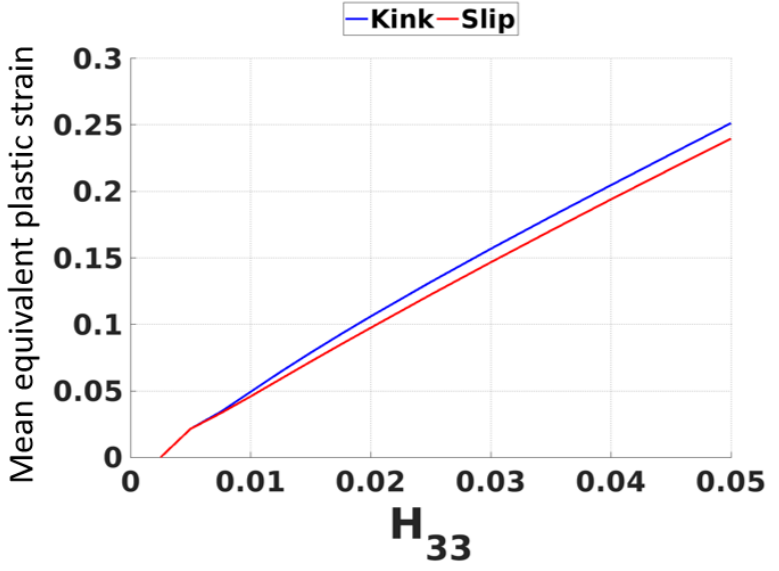

(b)

Figure 8: Evolution of slip/kink band mean equivalent plastic strain for the 2D (3 in-plane slip systems) (a) and a FCC 3D 64 grain polycrystal (b)

For geometrical reasons, the same amount of lattice rotation induces lower variation of Schmid factor in three dimensional simulations compared to the 2D simulations where all directions involved in Schmid factor calculations (glide direction, slip plane normal and loading directions) are coplanar. Hence, lattice rotation induced hardening should have a minor impact in 3D simulations than in 2D simulations, which is consistent with actual 210 results.

\subsection{Sensitivity to simulated Volume Element}

To our knowledge, kink bands have never been reported for strongly softening metals. However, the results presented in previous sections reveal large proportions of kinks in simulated localization band networks. In order to find out if these proportions are due to

the specific microstructure of the used unit cell, 9 random realizations of a 64 grain and one 512 grain 3D polycrystals have been generated to characterize the variability of simulated localization bands populations with volume element instances, and volume element size. Fig. 9 presents the evolution of slip/kink bands volume fraction with increasing loading. It is found that for the 64 grains volume element the standard deviation of simulated band volume fractions is generally smaller than the difference between slip and kink bands volume 


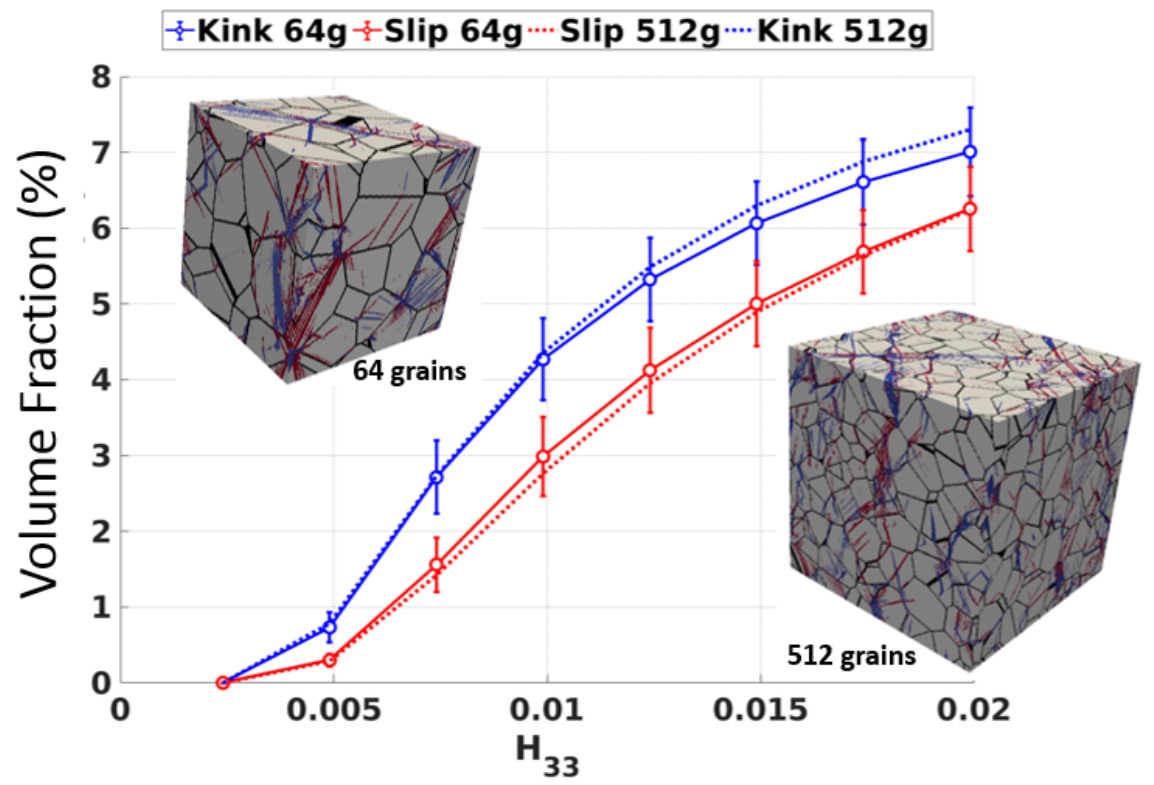

Figure 9: Slip/kink bands volume fraction evolution with loading in FCC polycrystal. Plotted value is the mean of 9 realizations of a 64 grains random Voronoi aggregate, error bars indicating standard deviation of the 9 simulations. Results for the 512 grains random Voronoi aggregate are plotted in dashed-lines. Associated localization maps show localization at $2 \%$ overall strain in one 64 grains polycrystal and in the 512 grains one.

fractions. Results of the 512 grain aggregate simulation are contained within the error bars, very close to the mean value of the 9x64 grains simulations. Besides, similar localization networks are observed on 64 and 512 grains simulations localization maps. It follows that a single 64 grains volume element may be sufficient to draw a qualitative analysis of slip/kink bands populations for these cubic crystals.

\section{Discussion : localization bands formation in crystal plasticity simulations}

Asaro and Rice [13] have shown that both slip and kink bands arise as the two possible bifurcation modes for elasto-plastic single crystals undergoing single slip at large strains. They defined this two modes as slip localization planes respectively orthogonal to glide plane normal direction $\boldsymbol{n}$ (slip bands) and glide direction $\boldsymbol{m}$ (kink bands). In the case of strain-softening crystals, their analysis shows that both modes become simultaneously 
possible at incipient plasticity. Incipient plasticity always occurs at small strain in metallic materials and in these conditions small strain formulation of crystal plasticity equations are valid to describe material behavior. In the small strain framework, resolved shear stress $\tau^{\alpha}$ on slip system $\alpha$ and plastic strain are computed as follow :

$\tau^{\alpha}=\boldsymbol{m} \cdot \boldsymbol{\sigma} \cdot \boldsymbol{n}$

$\dot{\varepsilon}_{p}=\sum \alpha \dot{\gamma}^{\alpha} \boldsymbol{m} \otimes_{\text {sym }} \boldsymbol{n}$

The Cauchy stress $\boldsymbol{\sigma}$ being a symmetric tensor and $\otimes_{\text {sym }}$ the symmetric tensor product, $\boldsymbol{m}$ and $\boldsymbol{n}$ play a perfectly symmetric role in Eq. 17: Inverting them leaves the equation unchanged. This consideration implies that kink and slip bifurcation modes are strictly equivalent at incipient plasticity with respect to the constitutive equations. It follows that structural effects, i.e. grain to grain plastic strain incompatibilities will govern the selection of slip or kink localization modes.

In order to provide an illustration of this property, two simulations have been carried out using the same grain geometry, grain orientations and material coefficients, but using two distinct crystal slip systems : the 12 FCC $\{111\}<110>$ slip systems and the 12 BCC $\{110\}<111>$ slip systems. The latter are indeed obtained by switching slip planes normals and glide directions of the FCC slip systems, thus according to Asaro and Rice analysis the potential localizations planes are the same in both polycrystals. The two identical microstructures induce identical structural effects and thus should activate the same localization planes at incipient plasticity. Simulations results clearly illustrate this point: In corresponding localization maps, shown on Fig. 10, most of FCC slip (resp. kink) bands have a kink (resp. slip) counterpart for the BCC crystal structure. Then, finite strain kinematics leads to distinct evolutions of slip and kink bands because of rotation induced hardening, discussed in previous sections. It explains why the two localization maps are not strictly equivalent after applying $1 \%$ overall tensile strain.

With a the view to investigating the influence of structural effects on slip/kink band formation a simulation has been carried out for a HCP crystal considering only the 3 basal slip systems $\{0001\}<1 \overline{2} 10>$. In that case the distribution of available localization planes 


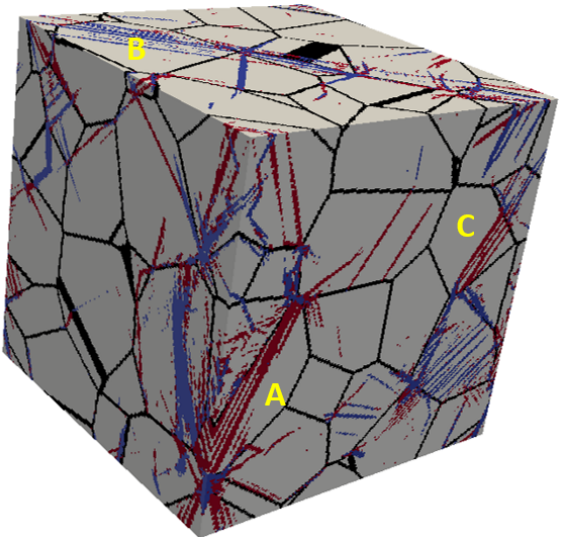

(a) FCC

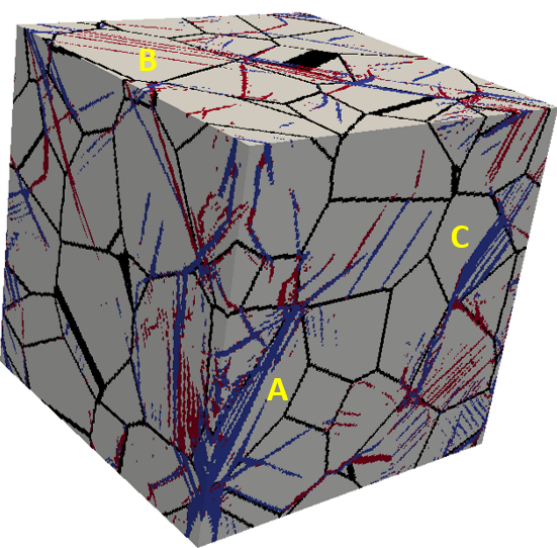

(b) $\mathrm{BCC}$

Figure 10: Localization maps of 3D polycrystals after loading to 1\% strain with (a) : 12 FCC slip systems $\{111\}[110]$ and (b) 12 BCC slip systems $\{110\}[111]$, with identical geometry and grains orientations. Grains marked A,B and C illustrate the almost identical band structure, with FCC slip banding (resp. kink) corresponding to BCC kink banding (resp. slip).

is then strongly anisotropic and should be more influenced by structural effects than in more isotropic cases such as cubic crystals. Indeed, the only potential plane for slip band formation in each grain is the basal plane, whereas kink banding, in prismatic planes offer 3 times more planes to accommodate grain to grain plastic incompatibilities. Fig. 11-a shows that kink bands volume fraction is approximately two times higher than slip band volume fraction, whereas the two quantities are much closer for the FCC crystals (Fig. 9). Associated localization map (Fig. 11-b) shows that localization occurs mainly at grain boundary triple lines from which kink bands seem to emerge more often than slip bands.

Triple lines grain boundary induce strong stress concentrations due to grain to grain incompatibilities. They are highly likely to trigger strain localization in some or all of the neighboring grains. Thus this structural effect will promote localization paths that extend over at least 2 grains and cross grain boundaries close to triple lines. Considering that geometrically there are three distinct kink planes against only one slip plane, the probability to form such transgranular localization paths across kink bands is higher. This could explain why the gap between slip and kink bands volume fractions is higher in the 


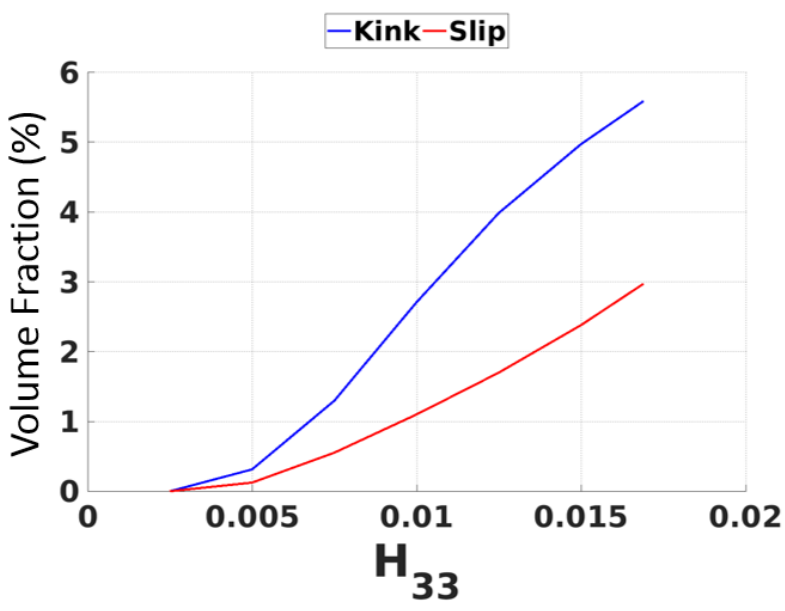

(a)

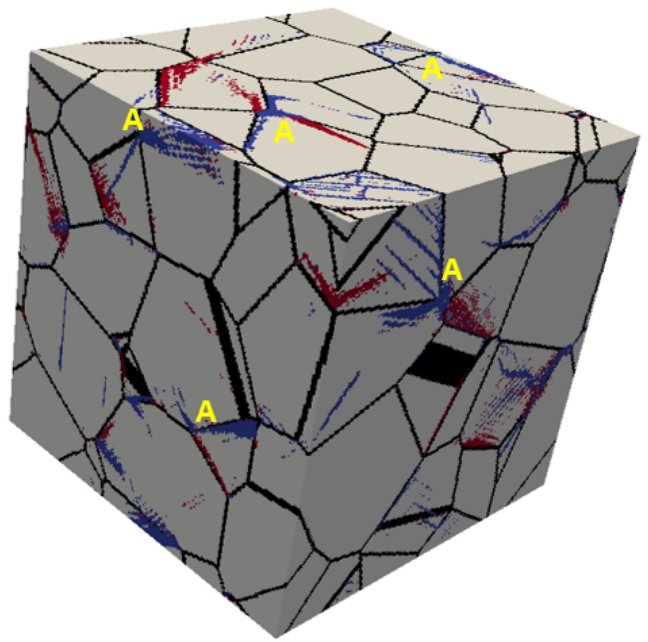

(b)

Figure 11: Slip/kink bands volume fraction evolution for a 64 grains random HCP polycrystal (a). Zones marked A in the associated localization map after $1 \%$ overall strain (b) show that localization occurs generally at triple grain boundary junctions mostly through kink banding.

case of the simulated HCP crystal.

Lebensohn et al. [15] carried out crystal plasticity simulations of ice HCP polycrystals deforming mainly through basal slip. As in our HCP simulation, they note significant occurrence of kink bands in their results which is in agreement with reported observations of kink bands in ice [4, 5]. Flouriot et al. [58] have also noted kink bands formation in crack-tip field simulations in a FCC crystal that are similar to those observed with crack-tip field measurements in ductile crystals [10]. As in this two situations, kink bands observations are mostly reported where strong strain incompatibilities arise such as crack-tip fields, compression of HCP single crystals [7] or deformation of strongly anisotropic HCP polycrystals [8, 4, 5] mainly deforming through basal slip. In those cases, crystal plasticity models appears to be well-suited to simulate their formation.

However, the case of irradiated Zirconium polycrystals provides a good example to also highlight their limitations. They are HCP crystals deforming mainly through basal slip and could exhibit formation of kink bands, like ice polycrystals, because of this slip anisotropy. On the contrary, deformed Zr polycrystal observations reveal only very intense slip bands, 
: active dislocation source
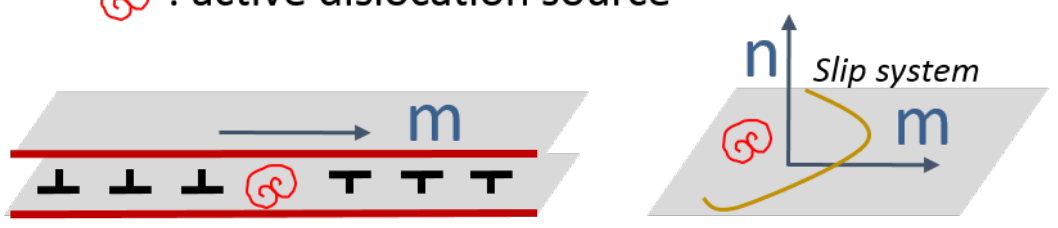

SLIP BAND

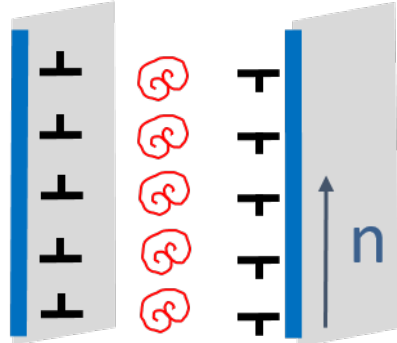

KINK BANDS

Figure 12: Schematics of active dislocation source distributions required to form a slip or a kink band. materials. 


\section{Conclusions}

We have used a massively parallel FFT solver to study intragranular strain localization induced by a strain softening classical crystal plasticity model. This numerical tool allows of complex crystal systems and achieve grid resolutions that allow for a fine description of intragranular localization bands. Our simulations show the formation of an intragranular localization network of narrow bands exhibiting high plastic slip.

We proposed a new method to study the nature of simulated localization bands based tion maps that where used to accurately identify the slip and kink band populations in the localization network, for both simple bidimensional simulations and realistic three dimensional polycrystals with complex crystal systems. Quantification of band volume fraction and mean plastic strain highlight the distinct evolution of slip and kink bands. Because of rotation induced hardening, kink bands tend to widen with increasing loading, and their volume fraction and mean strain level are less sensitive to mesh dependence and softening magnitude. We conclude that it is essential to identify the nature of localization modes when simulating intragranular strain localization.

In agreement with Asaro's and Rice's bifurcation analysis, we showed that slip or kink els. They only account for strain incompatibility effects on plastic localization mode formation, which translate into a large amount of kink bands in all simulations of softening polycrystals. This feature is in contradiction with experimental observation of localization bands in strongly softening metals which reveals the fundamental physical differences in slip or kink band formation from the dislocation mechanism perspective. Therefore, in order to accurately simulate intragranular strain localization at the continuum scale in softening metals, physical mechanisms that can promote individually kink or slip band formation need to be accounted for with more complex theories such as strain gradient plasticity. For instance, this framework allows to account in the constitutive equations for an energy density 
associated to the geometrically necessary dislocation (GND) density tensor, which is known to be high in kink bands because of the large lattice curvature that they induce. Such models could be able to reduce kink band formation and will be explored in future works.

\section{References}

[1] B. Jaoul, Etude de la plasticité et application aux métaux, Les Presses - Mines ParisTech, 1964.

[2] H. Neuhäuser, Dislocation in solids, ed. F.R.N. Nabarro. Holland Publishing Company Vol. 6, (1983) $319-440$.

[3] A. Churchman, The yield phenomena, kink bands and geometric softening in titanium crystals, Acta Metallurgica 3 (1955) 22-29. doi:10.1016/0001-6160(55)90006-7.

[4] C. Wilson, J. Burg, J. Mitchell, The origin of kinks in polycrystalline ice, Tectonophysics 127 (1986) 27-48. doi : 10.1016/0040-1951(86)90077-6.

[5] M. Montagnat, J. R. Blackford, S. Piazolo, L. Arnaud, R. A. Lebensohn, Measurements and fullfield predictions of deformation heterogeneities in ice, Earth and Planetary Science Letters 305 (2011) 153-160. doi:10.1016/j.epsl.2011.02.050.

[6] P. Mansuy, A. Philip, J. Meyssonier, Localization of deformation in polycrystalline ice, J. Phys. 11 (2001) 267-274. doi:http://dx.doi.org/10.1051/jp4:2001433.

[7] K. Hagihara, T. Mayama, M. Honnami, M. Yamasaki, H. Izuno, T. Okamoto, T. Ohashi, T. Nakano, Y. Kawamura, Orientation dependence of the deformation kink band formation behavior in Zn single crystals, International Journal of Plasticity 77 (2016) 174-191. doi:10.1016/j.ijplas .2015.10.005.

[8] K. Hagihara, T. Okamoto, M. Yamasaki, Y. Kawamura, T. Nakano, Electron backscatter diffraction pattern analysis of the deformation band formed in the Mg-based long-period stacking ordered phase, Scripta Materialia 117 (2016) 32-36. doi:10.1016/j.scriptamat.2016.02.016.

[9] W. Crone, T. Shield, Experimental study of the deformation near a notch tip in copper and copperberyllium single crystals, Journal of the Mechanics and Physics of Solids 49 (2001) 2819-2938. doi: https://doi.org/10.1016/S0022-5096(01)00080-1.

[10] J. W. Kysar, C. L. Briant, Crack tip deformation fields in ductile single crystals, Acta Materialia 50 (2002) 2367-2380. doi:10.1016/S1359-6454(02)00070-8.

[11] S. D. Patil, R. Narasimhana, R. K. Mishra, Observation of kink shear bands in an aluminium single crystal fracture specimen, Scripta MAterialia 61 (2009) 465-468. doi:10.1016/j.scriptamat. 2009. 04.043.

[12] Y. Zheng, W. Zeng, Y. Wang, S. Zhang, Kink deformation in a beta titanium alloy at high strain rate, Material Science \& Engineering A 702 (2017) 218-224. doi:10.1016/j.msea.2017.07.015. 
[13] R. Asaro, J. Rice, Strain localization in ductile single crystals, Journal of the Mechanics and Physics of Solids 25 (1977) 309-338. doi:10.1016/0022-5096(77)90001-1.

[14] S. Forest, Modeling slip, kink and shear banding in classical and generalized single crystal plasticity, Acta Materialia 46 (1998) 3265-3281. doi:10.1016/S1359-6454(98)00012-3.

[15] R. Lebensohn, M. Montagnat, P. Mansuy, P. Duval, J. Meysonnier, A. Philip, Modeling viscoplastic behavior and heterogeneous intracrystalline deformation of columnar ice polycrystals, Acta Materialia 57 (2009) 1405-1415. doi:10.1016/j.actamat.2008.10.057.

[16] Y. Kimura, R. Ueta, K. Shizawa, Dislocation-based crystal plasticity FE analysis for kink band formation in Mg-based LPSO phase considering higher-order stress, Procedia Manufacturing 15 (2018) 1825-1832. doi:10.1016/j.promfg.2018.07.208.

[17] Y. Estrin, L. Kubin, Local strain hardening and nonuniformity of plastic deformation, Acta Metallurgica 34 (1986) 2455-2464. doi:https://doi.org/10.1016/0001-6160(86)90148-3.

[18] Y. Brechet, G. Canova, L. Kubin, Static versus propagative strain localisations, Scripta Metallurgica et Materialia 29 (1993) 1165-1170. doi:10.1016/0956-716X(93)90103-Y.

[19] D. Ulmer, C. Altstetter, Hydrogen-induced strain localization and failure of austenitic stainless steels at high hydrogen concentrations, Acta Metallurgica et Materialia 39 (1991) 1237-1248. doi:https: //doi.org/10.1016/0956-7151(91)90211-I.

[20] I. Aubert, N. Saintier, J. Olive, Crystal plasticity computation and atomic force microscopy analysis of the internal hydrogen-induced slip localization on polycrystalline stainless steel, Scripta Materialia 66 (2012) 698-701. doi:10.1016/j.scriptamat.2012.01.019.

[21] J. Sharp, Correlation between cleared channels and surface slip steps in neutron irradiated copper crystals, Radiation Effects 14 (1972) 71-75. doi:10.1080/00337577208230474.

[22] D. Edwards, B. Singh, J. Bilde-Sorensen, Initiation and propagation of cleared channels in neutronirradiated pure copper and a precipitation hardened CuCrZr alloy, Journal of Nuclear Materials 342 (2005) 164-178. doi:10.1016/j.jnucmat.2005.04.001.

[23] F. Onimus, I. Monnet, J. Béchade, C. Prioul, P. Pilvin, A statistical TEM investigation of dislocation channeling mechanism in neutron irradiated zirconium alloys, Journal of Nuclear Materials 328 (2004) 165-179. doi:10.1016/j.jnucmat.2004.04.337.

[24] L. Fournier, A. Serres, Q. Auzoux, D. Leboulch, G. Was, Proton irradiation effect on microstructure, strain localization and iodine-induced stress corrosion cracking in Zircaloy-4, Journal of Nuclear Materials 384 (2009) 38-47. doi:10.1016/j.jnucmat.2008.10.001.

[25] M. Sauzay, K. Bavard, W. Karlsen, TEM observations and finite element modelling of channel deformation in pre-irradiated austenitic stainless steels - interactions with free surfaces and grain boundaries, Journal of Nuclear Materials 406 (2010) 152-165. doi:10.1016/j.jnucmat.2010.01.027. 
[26] T. Byun, N. Hashimoto, K. Farrell, E. Lee, Characteristics of microscopic strain localization in irradiated 316 stainless steels and pure vanadium, Journal of Nuclear Materials 349 (2006) 251-264. doi:10.1016/j.jnucmat.2005.10.011.

[27] T. Byun, N. Hashimoto, K. Farell, Deformation mode map of irradiated 316 stainless steel in true stressdose space, Journal of Nuclear Materials 351 (2006) 303-315. doi:10.1016/j.jnucmat.2006.02.033.

[28] K. Field, M. N. Gussev, J. Busby, Microstructural characterization of deformation localization at small strains in a neutron-irradiated 304 stainless steel, Journal of Nuclear Materials 452 (2014) 500-508. doi:10.1016/j.jnucmat.2014.05.053.

[29] N. Hashimoto, T. Byun, K. Farell, S. Zinkle, Deformation microstructure of neutron-irradiated pure polycrystalline vanadium, Journal of Nuclear Materials 336 (2005) 225-232. doi :10.1016/j.jnucmat. 2004.09 .017$.

[30] T. Mori, M. Meshii, Plastic deformation of quench-hardened aluminium single crystals, Acta Metallurgica 17 (1969) 167-175. doi:10.1016/0001-6160(69)90137-0.

[31] M. Bapna, M. Meshii, Deformation of quench-hardened gold crystals, Materials Science and Engineering 16 (1974) 181-191. doi:https://doi.org/10.1016/0025-5416(74)90152-9.

[32] F. Onimus, L. Dupuy, F. Mompiou, In situ TEM observations of interactions between gliding dislocations and prismatic loops in Zr-ion irradiated zirconium alloys, Progress in Nuclear Energy 57 (2012) 77-85. doi:10.1016/j.pnucene.2011.10.005.

[33] M. Lai, C. Tasan, D. Raabe, Deformation mechanism of omega-enriched Ti-Nb-based gum metal

: Dislocation channeling and deformation induced omega-beta transformation, Acta Materialia 100 (2015) 290-300. doi:http://dx.doi.org/10.1016/j.actamat.2015.08.047.

[34] D. Rodney, Atomic-scale modeling of clear band formation in FCC metals, Nuclear Instruments and Methods in Physics Research B 225 (2005) 100-110. doi:10.1016/j.nimb.2004.10.029.

[35] T. Nogaret, D. Rodney, M. Fivel, C. Robertson, Clear-band formation simulated by dislocation dy415 namics: Role of helical turns and pile-ups, Journal of Nuclear Materials 380 (2008) 22-29. doi: $10.1016 / j \cdot j n u c m a t .2008 .07 .001$.

[36] A. Arsenlis, M. Rhee, G. Hommes, R. Cook, J. Marian, A dislocation dynamics study of the transition from homogeneous to heterogeneous deformation in irradiated body-centered cubic iron, Acta Materialia 60 (2012) 3748-3757. doi:10.1016/j.actamat.2012.03.041.

[37] A. Arsenlis, B. D. Wirth, M. Rhee, Dislocation density-based constitutive model for the mechanical behaviour of irradiated Cu, Philosophical Magazine 84:34 (2004) 3617-3635. doi:10.1080/ 14786430412331293531.

[38] S. Krishna, A. Zamiri, S. De, Dislocation and defect density-based micromechanical modeling of the mechanical behavior of FCC metals under neutron irradiation, Philosophical Magazine 90:30 (2010) 
4013-4025. doi:10.1080/14786435.2010.502150.

[39] F. Onimus, J.-L. Bechade, A polycristalline modeling of the mechanical behavior of neutron irradiated zirconium alloys, Journal of Nuclear Materials 384 (2009) 163-174. doi:10.1016/j.jnucmat.2008. 11.006

[40] N. Barton, A. Arsenlis, J. Marian, A polycrystal plasticity model of strain localization in irradiated iron, Journal of the Mechanics and Physics of Solids 61 (2013) 341-351. doi:10.1016/j .jmps .2012.10.009.

[41] A. Patra, D. McDowell, Crystal plasticity-based constitutive modelling of irradiated BCC structures, Philosophical Magazine 92:7 (2012) 861-887. doi:10.1080/14786435.2011.634855.

[42] X. Xiao, D. Song, J. Xue, H. Chu, H. Duan, A size-dependent tensorial plasticity model for FCC single crystals with irradiation, International Journal of Plasticity 65 (2015) 152-167. doi:10.1016/ j.ijplas.2014.09.004.

[43] J. Hure, S. E. Shawish, L. Cizelj, B. Tanguy, Intergranular stress distributions in polycrystalline aggregates, Journal of Nuclear Materials 476 (2016) 231-242. doi:10.1016/j.jnucmat.2016.04.017.

[44] M. Zhang, F. Bridier, P. Villechaise, J. Mendez, D. McDowell, Simulation of slip band evolution in duplex Ti-6Al-4V, Acta Materialia 58 (2010) 1087-1096. doi:10.1016/j .actamat.2009.10.025.

[45] T. Erinosho, F. Dunne, Strain localization and failure in irradiated zircaloy with crystal plasticity, International Journal of Plasticity 71 (2015) 170-194. doi:10.1016/j.ijplas.2015.05.008.

[46] A. Patra, D. McDowell, Continuum modelling of localized deformation in irradiated bcc materials, Journal of Nuclear Materials 432 (2013) 414-427. doi:10.1016/j.jnucmat.2012.08.021.

[47] A. Patra, D. McDowell, Crystal plasticity investigation of the microstructural factors influencing dislocation channeling in a model irradiated bcc material, Acta Materiala 110 (2016) 364-376. doi: $10.1016 / j$.actamat .2016 .03 .041$.

[48] J. Mandel, Equations constitutives et directeurs dans les milieux plastiques et viscoplastiques, International Journal of Solids and Structures 9 (1973) 725-740. doi:10.1016/0020-7683 (73)90120-0.

[49] C. Ling, B. Tanguy, J. Besson, S. Forest, F. Latourte, Void growth and coalescence in triaxial stress fields in irradiated FCC single crystals, Journal of Nuclear Materials 492 (2017) 157-170. doi:10. 1016/j.jnucmat.2017.04.013.

[50] H. Moulinec, P. Suquet, A numerical method for computing the overall response of nonlinear composites with complex microstructure, Comput. Methods Appl. Mech. Engrg. 157 (1998) 69-94. doi:10.1016/ S0045-7825 (97)00218-1.

[51] F. Willot, Fourier-based schemes for computing the mechanical response of composites with accurate local fields, Comptes Rendus Mécanique 343 (2015) 232-245. doi :https://doi .org/10.1016/j . crme. 2014.12 .005

[52] M. Schneider, D. Merkert, M. Kabel, FFT-based homogenization for microstructures discretized by 
linear hexahedral elements, International journal for numerical methods in engineering 109 (2017) 1461-1489. doi:10.1002/nme.5336.

[53] D. Anderson, Iterative procedures for nonlinear integral equations, J. Assoc. Comput. Mach. 12 (1965) 547-560. doi:10.1145/321296.321305.

[54] H. Walker, P. Ni, Anderson acceleration for fixed-point iterations, SIAM J. Numer Anal. 49 (2011) 1715-1735. doi:https://doi.org/10.1137/10078356X.

[55] I. Ramière, T. Helfer, Iterative residual-based vector methods to accelerate fixed point iterations, Computer and Mathematics with Applications 70 (2015) 2210-226. doi:https://doi.org/10.1016/ j. camwa.2015.08.025.

[56] T. Helfer, B. Michel, J.-M. Proix, M. Salvo, J. Sercombe, Introducing the open-source MFront code generator: Application to mechanical behaviours and material knowledge management within the PLEIADES fuel element modelling platform, Computer and Mathematics with Applications 70 (2015) 994-1023. doi:https://doi.org/10.1016/j.camwa.2015.06.027.

[57] C. Ling, B. Tanguy, J. Besson, S. Forest, F. Latourte, E. Bosso, An elastoviscoplastic model for porous single crystals at finite strains and its assessment based on unit cell simulations, International Journal of Plasticity 84 (2016) 58-87. doi:https://doi.org/10.1016/j.ijplas.2016.05.001.

[58] S. Flouriot, S. Forest, G. Cailletaud, A. Köster, L. Rémy, B. Burgardt, V. Gros, S. Mosset, J. Delautre, Strain localization at the crack tip in single crystal CT specimens under monotonous loading: 3D finite element analyses and application to nickel-base superalloys, International Journal of Fracture 124 (2003) 44-77. doi:10.1023/B:FRAC.0000009300 .70477.ba. 
Supplementary Material
Click here to download Supplementary Material: Film_localisation_3D.avi

Supplementary Material
Click here to download Supplementary Material: Film_localisation_3D.avi 

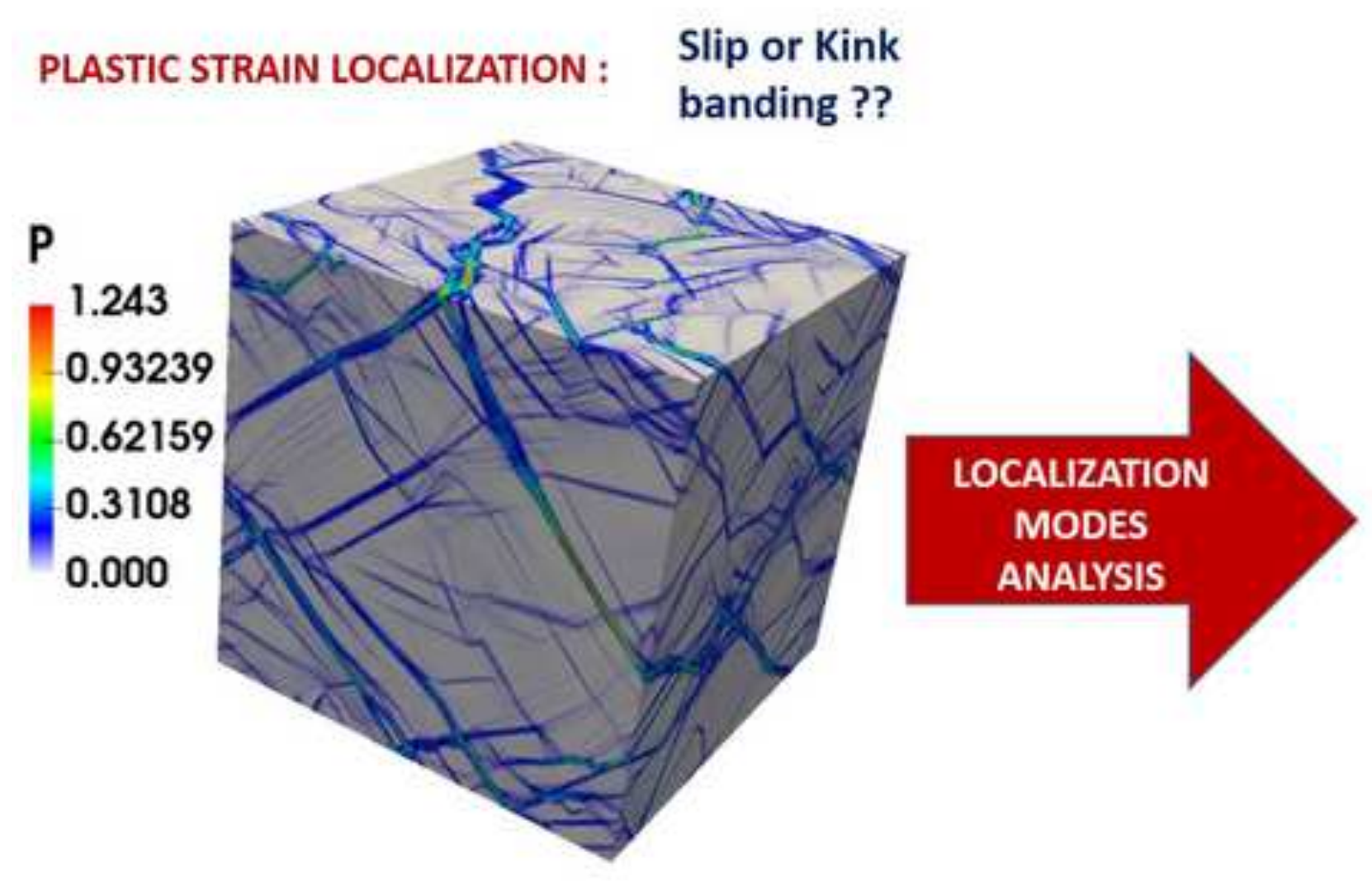

Slip / Kink bands maps
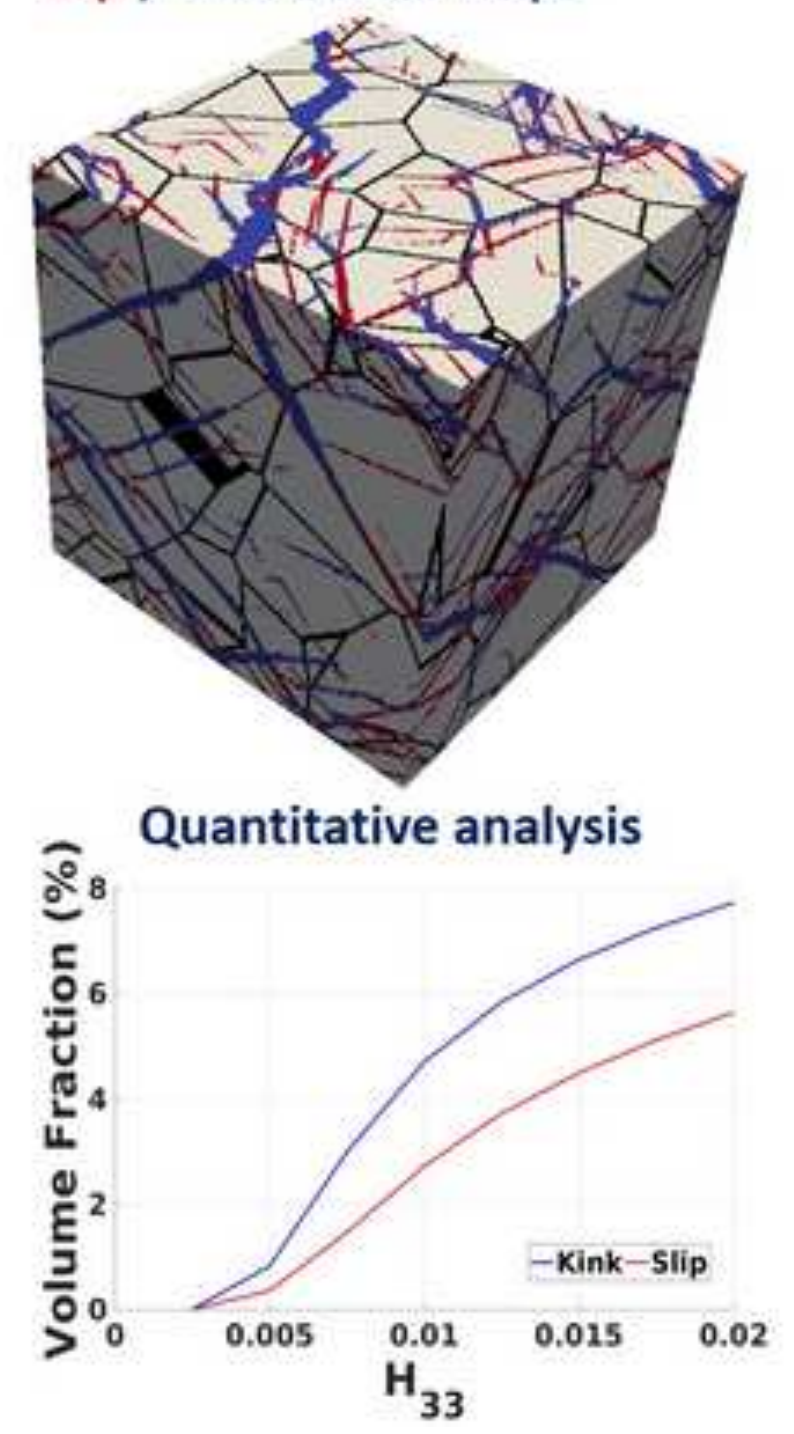